\title{
Application of Linear Prediction, Self-Adaptive Noise Cancellation and Spectral Kurtosis in Identifying Natural Damage of a Rolling Element Bearing in a Gearbox
}

\author{
Cristóbal Ruiz-Cárcel, Enrique Hernani-Ros, Yi Cao, Michael Corsar and David Mba \\ School of Engineering, Cranfield University (UK); Building 52, Cranfield University, Bedfordshire, MK43 OAL, \\ $U K$
}

\author{
Pramesh Chandra \\ Moog Aircraft Group, Wolverhampton (UK), Wobaston Road, Wolverhampton, WV9 5EW, UK
}

(Received 20 September 2012; revised 17 December 2013; accepted 26 February 2014)

\begin{abstract}
The ability to detect and diagnose faults in rolling element bearings is crucial for modern maintenance schemes. Several techniques have been developed to improve the ability of fault detection in bearings using vibration monitoring, especially in those cases where the vibration signal is contaminated by background noise. Linear Prediction and Self-Adaptive Noise Cancellation are techniques which can substantially improve the signal to noise ratio of the signal, improving the visibility of the important signal components in the frequency spectrum. Spectral Kurtosis has been shown to improve bearing defect identification by focusing on the frequency band with a high level of impulsiveness. In this paper the ability of these three methods to detect a bearing fault is compared using vibrational data from a specially designed test rig that allowed fast natural degradation of the bearing. The results obtained show that the Spectral Kurtosis was able to detect an incipient fault in the outer race of the bearing much earlier than any other technique.
\end{abstract}

\section{NOMENCLATURE}

\begin{tabular}{ll}
$a(k)$ & Weight attached to each observation in LP \\
ANC & Adaptive Noise Cancelling \\
$e$ & Output in ANC and SANC \\
$f$ & Frequency \\
GM & Gear mesh frequency \\
$H$ & Filter length \\
IRD & Inner race defect frequency \\
$K$ & Kurtosis \\
$L F$ & Line Frequency \\
$L P$ & Linear prediction \\
$n$ & Time point \\
$N$ & Number of past samples considered in the \\
& calculation of $R_{\tau}$ \\
$n_{0}$ & Reference noise \\
$n_{1}$ & Uncorrelated eference noise \\
ORD & Outer race defect frequency \\
$p$ & Number of past samples considered in $L P$ \\
$R_{\tau}$ & Autocorrelation function \\
$S$ & Signal of interest in ANC/SANC \\
$S A N$ & Self-Adaptive Noise Cancellation \\
$S K$ & Spectral Kurtosis \\
$S N R$ & Signal to Noise Ratio \\
$S S$ & Shaft speed frequency \\
$W$ & Vector of filter coefficients \\
$w$ & Filter weights in ANC and SANC \\
$x$ & Random signal \\
$\hat{x}(n)$ & Predictable part of signal $x$ at \\
$y(n)$ & Filter output \\
\hline
\end{tabular}

$\begin{array}{ll}\Delta & \text { Time delay } \\ \Delta f & \text { Frequency band width } \\ \mu & \text { Forgerring factor } \\ \mu & \text { Average value } \\ \sigma & \text { Standard deviation }\end{array}$

\section{INTRODUCTION}

Rolling element bearings are important components in rotating machinery. By monitoring the vibration signature of bearings, it is possible to obtain important information about their condition and use this information to improve the maintenance strategy. Diagnostic techniques based on vibration are mainly concerned with the extraction of defect features in the acquired signal, which can be related to the healthy or defective state of vital parts in a machine. Many different diagnostic methods have been successfully used to identify machine faults, processing the vibration signal in the time or frequency domain, in order to locate and quantify any existing damage. In complex machines the signal acquired is normally inclusive of additive background noise from other machine components or subsystems, which can make it difficult or sometimes impossible to identify the fault patterns in the signal.

In the case of bearings, the fault is produced typically by the damage of the surface of the inner or outer race or the rolling elements. When a damaged surface contacts another rolling surface, a force impulse is generated, which excites resonances in the bearing and the machine. ${ }^{1}$ The successive impacts generate a vibration signal, which often has an impulsive repeti- 